\title{
Chapter 6 \\ Geosmartness for Personalized and Sustainable Future Urban Mobility
}

\author{
Martin Raubal, Dominik Bucher, and Henry Martin
}

\begin{abstract}
Urban mobility and the transport of people have been increasing in volume inexorably for decades. Despite the advantages and opportunities mobility has brought to our society, there are also severe drawbacks such as the transport sector's role as one of the main contributors to greenhouse-gas emissions and traffic jams. In the future, an increasing number of people will be living in large urban settings, and therefore, these problems must be solved to assure livable environments. The rapid progress of information and communication, and geographic information technologies, has paved the way for urban informatics and smart cities, which allow for large-scale urban analytics as well as supporting people in their complex mobile decision making. This chapter demonstrates how geosmartness, a combination of novel spatial-data sources, computational methods, and geospatial technologies, provides opportunities for scientists to perform large-scale spatio-temporal analyses of mobility patterns as well as to investigate people's mobile decision making. Mobility-pattern analysis is necessary for evaluating real-time situations and for making predictions regarding future states. These analyses can also help detect behavioral changes, such as the impact of people's travel habits or novel travel options, possibly leading to more sustainable forms of transport. Mobile technologies provide novel ways of user support. Examples cover movement-data analysis within the context of multi-modal and energy-efficient mobility, as well as mobile decision-making support through gaze-based interaction.
\end{abstract}

M. Raubal (凶) $\cdot$ D. Bucher $\cdot$ H. Martin

Institute of Cartography and Geoinformation, ETH Zurich, Zurich, Switzerland

e-mail:mraubal@ethz.ch

D. Bucher

e-mail: dobucher@ethz.ch

H. Martin

e-mail: martinhe@ethz.ch 


\subsection{Introduction}

Urban mobility and the transport of people have been rising inexorably for decades. Despite the many advantages and opportunities, mobility has brought to our society, there are also severe drawbacks such as the transport sector's role as one of the main contributors to $\mathrm{CO}_{2}$ emissions, traffic jams, and mass event catastrophes (Elliott and Urry 2010; Taaffe et al. 1996). Forecasts show that by 2030, the world will have 41 megacities each with more than 10 million inhabitants (UN 2014), and by the year 2050, approximately $80 \%$ of the European population will be living in urban areas (Caragliu et al. 2011). Therefore, these challenging problems must be solved to assure livable environments for future generations.

The rapid progress of information and communication technologies (ICT) and geographic information technologies has paved the way for urban informatics and smart cities, which allow for large-scale urban analytics as well as supporting people in their complex mobile decision making. This chapter demonstrates how geosmartness, a combination of novel spatial-data sources, computational methods, and geospatial technologies, provides ample opportunities for scientists to perform largescale spatio-temporal analyses of mobility patterns as well as investigate people's mobile decision making. This application of novel methods and technologies with spatial big data will allow for unprecedented possibilities of evaluating current states of urban systems including their citizens in real time, and making predictions and forecasts of future states.

Mobility-pattern analysis is necessary for evaluating real-time situations but also for making short- and longer-term predictions regarding the transportation network. In addition, these analyses can help detect behavioral changes, such as the impact of people's travel habits or novel travel options, possibly leading to more sustainable forms of transport. Sustainable urban mobility will become ever more important in order to curb greenhouse-gas emissions in the future. Long-term decarbonization of transport will not solely be achievable through new technology, such as vehicle efficiency measures, powertrain technology, and new energy carriers, but will require people's efforts in containing demand and shifting to lower-emission transport modes (Boulouchos et al. 2017).

Mobile technologies help to identify individual-oriented problems and provide novel ways of personalized user support. Spatial Big Data can be utilized to support people in their location-based decision making, in combination with novel technologies and interaction concepts, such as location-based services and gaze-based interaction. This will lead to more effective and efficient spatio-temporal decision making, and, hopefully, contribute to sustainable urban mobility of the future.

This chapter starts by introducing geosmartness and its major enablers, namely geospatial technologies, spatial, big data and spatio-temporal computational methods. We then investigate the analysis of urban-mobility patterns, including data, prediction, and labeling methods. The section is complemented by an overview of mobility studies and a detailed example focusing on multi-modal and energyefficient mobility. In the next section, we elaborate on the potential of geospatial 


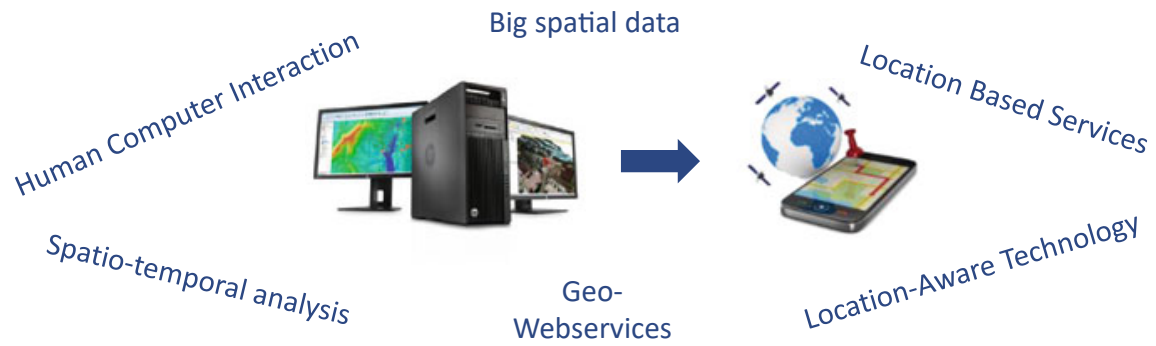

Fig. 6.1 Methods and tools enabling geosmartness

and persuasive technologies to support people in sustainable mobility. This includes motivational aspects and methods for detecting and supporting behavioral change. The section also includes an overview of studies in this area and the description of a recent study targeting the change of mobility behavior. In the penultimate section, we explain the specificities of mobile decision making, introduce the technique of mobile eye-tracking and the concept of gaze-based interaction, and demonstrate how their combination can enable personalized gaze-based decision support. The final section presents conclusions and directions for future work.

\subsection{Geosmartness}

Geosmartness relates to the vast opportunities of utilizing novel geospatial technologies, spatial big data, and spatio-temporal computational methods for solving many of the world's challenging problems in the domains of mobility, transport, and climate. It has been made possible through the rapid progress of computing, communication, and information technologies, but also by theoretical advancements in fields such as geographic information science (or to be more encompassing, spatial data science including its representations, models, and analysis methods) (Goodchild 1992; Raubal 2019; Reitsma 2012).

Geosmartness is essential for successfully transforming traditional cities and urban areas into smart cities, which are in essence digitally integrated urban spaces based on a real-time sensor-based control system. Such a system comprises technology, people, and community (Nam and Pardo 2011), and its major goal and challenge is to solve key problems of growing cities through integration of technology and environment (Batty et al. 2012). Ratti and Claudel (2016) provide an overview of future smart-city concepts, emphasizing also the value of open data and platforms, and the necessity for smart citizens. Concrete efforts and lessons learned when building a smart city have been demonstrated and described, such as for Barcelona (Gasco-Hernandez 2018).

The various methods and tools enabling geosmartness (Fig. 6.1) cover the traditional stages of a GIS (geographic information system) process, including spatial data 
modeling, representation, analysis, and presentation (Longley et al. 2011), but on a much wider scale, involving novel interfaces, cutting-edge information technology, and real-time sensor data (not only at the geographic scale; Montello 1993).

Spatial big data results from the ever-increasing progress in computing, communication, and information technologies. They come in the form of massive movementtrajectory datasets, fine-resolution environmental data, or specific user-behavior data (e.g., from eye-tracking), often in real time. Li et al. (2016) characterize geospatial big data by the following dimensions:

- Volume: Exabytes (or more) of imagery, sensor, and location-based social-media data raise both storage and analysis issues.

- Variety: relating to the various types of geospatial data, such as raster, vector, network, structured, and unstructured data and their integration.

- Velocity: Real-time trajectory and social-media data, and other continuous streams of sensor data require data processing at the same speed as data acquisition.

- Veracity: Depending on the sources, geospatial big data vary in accuracy and precision, and impact reliability and trust. Quality assessment may therefore be difficult.

- Visualization: on the one hand providing procedures to impose human reasoning on big data analysis, and on the other hand facilitating the communication of patterns and relationships as the results from such analysis.

- Visibility: Geospatial big data can nowadays be efficiently accessed and processed through cloud-computing technologies.

In order to pursue knowledge discovery from these complex and massive spatial data, traditional spatio-temporal analysis methods are now extended and complemented on a large scale by machine-learning approaches (Raubal et al. 2018). Machine learning is applied to spatial big data in CyberGIS analytics, for spatiotemporal outlier and anomaly detection, and for predicting human spatial behavior. Spatial data science enhances machine learning by proposing methods for spatiotemporal modeling and context integration to achieve better results and higher performance. In the area of mobility and transport, it has recently been demonstrated how graph convolutional neural networks (GCNs) can be used for imputing human activity purposes from GPS trajectory data (Martin et al. 2018). Multiple personalized graphs were utilized to model human mobility behavior and to embed a large variety of spatio-temporal information and structure in the graphs' weights and connections. These graphs served as input to the GCNs, which in turn exploited such structure.

Geographic information technologies encompass systems and services that exploit geoinformation to support people's spatio-temporal decision making (Raubal 2018). They utilize data related to locations in space and time, and process these data with respect to spatial locations, which results in increased complexity during reasoning and data analysis. Nowadays, geographic information technologies not only include desktop GIS for acquiring, representing, analyzing, and visualizing spatio-temporal data, but also location-based services (LBS), which support people in their mobile decision-making by providing spatial information based on their current locations, 
typically by relying on GPS (Global Positioning System) technology built into them (Brimicombe and Li 2009). LBS can be further enhanced by other context information, such as the user's gaze. This allows taking the user's viewing direction into account (Anagnostopoulos et al. 2017), leading, for example, to personalized audio guides that help users to find objects in the environment, and adapting the audio content to what has previously been looked at (Kwok et al. 2019). This directly relates to geographic human-computer interaction, i.e., people's interaction with geographic information technologies (Hecht et al. 2011). Novel interaction modalities and paradigms, and context-aware user interfaces, are available nowadays. In addition to traditional user interfaces through which people can interact with textbased information or cartographic maps, novel interaction modes, such as audio, gesture, gaze, or vibration (Gkonos et al. 2017), and displays integrating augmented and virtual reality exist (Rudi et al. 2016).

\subsection{Analyzing Urban-Mobility Patterns}

Mobility has always been a crucial part of urban life. As cities grow larger, moving millions of people for work, errands, or leisure activities becomes increasingly complicated, and when unmanaged, mobility has severe negative effects such as greenhouse-gas emissions, air pollution, health problems (Krzyżanowski et al. 2005), and traffic congestion.

To mitigate these negative effects, system-level actions must be combined with actions that empower mobility behavior change of individuals (Banister 2011). Examples for system-level interventions are the implementation of smart traffic management systems, or adaptive and attractive public transport systems. Individual mobility change may be achieved by enabling new forms of mobility, such as mobility as a service (MaaS), on-the-fly ride sharing or on-demand last-mile buses. These novel mobility concepts are all manifestations of geosmartness as they are ways to optimally allocate spatial resources, for which they require detailed knowledge of individual and aggregated city-wide mobility behavior.

\subsubsection{Data}

With the proceeding digitalization of our society, cities have become a melting pot for data from many different sources. This development bears new and unprecedented potential of gaining detailed knowledge about people's mobility behavior that can be used to enable sustainable mobility concepts. From the perspective of movement analysis, all available data can be divided into two groups: tracking data and context data.

Quantitative movement analysis is based on tracking data, which can be described as sequentially recorded and time-stamped locations. In the past, the elicitation of 
these data was based on paper or telephone surveys, but over the past decade the diversity of tracking-data sources multiplied and today, a manifold of different types of tracking data are available. Examples are global navigation satellite system (GNSS) tracking data (Zheng et al. 2008), location data based on the proximity to WiFi hotspots (Sapiezynski et al. 2015), location data from social networks (Hasan et al. 2013), public transport smart card data (Zhong et al. 2016), call detail record (CDR) data (González et al. 2008; Yuan and Raubal 2016b; Yuan et al. 2012), and credit-card transactions (Clemente et al. 2018).

These sources offer new possibilities to analyze movement within cities. However, the many possibilities to record urban movement create a heterogeneous landscape of tracking data sets. Four factors are particularly important when comparing different data sets:

- Tracking style (e.g., fixed versus moving tracking devices as in the Eulerian versus Lagrangian tracking style concept; Laube 2014)

- Spatio-temporal resolution (i.e., sampling rate)

- Spatio-temporal distribution (track point distribution, e.g., regular vs. burst patterns)

- Sample biases (e.g., daily urban mobility vs. mobility of tourists).

Due to these differences, it is difficult to compare results across different data sets and to develop data-agnostic methods. These are still open research challenges to be addressed in the near future in order to ensure the success of urban movement data analytics.

The second part of the data that are available in an urban setting does not describe the movement of people itself but the context in which people are moving. These context data are important for the analysis of human mobility patterns because human movement is always set in and influenced by its spatio-temporal context (Sharif and Alesheikh 2018). For example, when driving, our movement is restricted by the street network, when using public transportation, we depend on fixed schedules; we walk faster when it rains (Knoblauch et al. 1996), and we move differently depending on the urban or suburban setting (Yuan and Raubal 2016a).

In the past, only a few sources of context data, usually with a coarse spatiotemporal resolution, were available. This changed with progress in the digitalization of cities, and today many different context data sources with fine spatio-temporal resolution are available. Among the most important ones, urban movement analytics are volunteered geographic information (VGI) platforms such as OpenStreetMap, which provides easy access to road networks and point-of-interest data. A more recent trend inspired by the success of the open-data community is the open-data movement at the city level. Today many cities have open-data policies and publish their data on open-data platforms. Sensor networks provide another important source for context data, such as temperature, noise, pedestrian counts, or air quality. Examples for sensor networks with publicly available data are VGI-based platforms such as OpenSenseMap or luft-daten.info for air-quality data. There are also sensor networks operated by the cities themselves such as the Array of Things project in Chicago. Other context data include photogrammetry or street imagery data such as Google 
Street View. The latter has been used to automatically assess the well-being of neighborhoods (Suel et al. 2019) and to develop image-based navigation systems (Mirowski et al. 2019).

\subsubsection{Computational Methods for Large-Scale Spatio-temporal Mobility-Pattern Analysis}

Movement and context data generated by smart cities offer unprecedented possibilities for analyzing urban-mobility patterns (see also Chaps. 28 and 29). However, the large data volume, the variety of the new urban data sources, and the large bandwidth of tasks require the enhancement of traditional GIS methods known from classical movement analytics (Long and Nelson 2013; Zheng 2015).

\subsubsection{Data Preparation and Data Fusion}

Especially for the preparation of the data and for the combination of different spatial datasets, well-established GIS methods are of great importance. Important preprocessing steps are GPS-trajectory segmentation, map matching, spatial filtering or movement-trajectory compression. In the same way, proven GIS methods can be used to combine different spatial datasets and to enrich trajectories with context data (Jonietz and Bucher 2017).

However, with the growing data volume, manual processing will not be an option in the future. Therefore, scalability of workflows must always be kept in mind. This includes the choice of efficient algorithms, their efficient implementation, and the possibility of processing using distributed frameworks (e.g., big data frameworks).

\subsubsection{Prediction and Labeling}

The following tasks are of great importance when analyzing urban-mobility patterns: adding semantic information to unlabeled data and predicting urban mobility for a short forecast horizon (e.g., hours or days).

Adding semantic information is important because even though digital cities provide large volumes of data, large-scale tracking data sets are usually recorded passively (e.g., without interaction of the user) and are therefore unlabeled (Bauer et al. 2016). In order to interpret and understand urban mobility, these datasets must be enriched with semantic information such as activity labels or mode of transport.

The prediction of movement and mobility is important to optimize future states of the mobility system and to create flexible and personalized mobility offers. Knowing the future mobility demand within a city allows for optimizing the schedule of public transport systems, taxi placements, or timings of traffic lights. On the other hand, 
knowing about the subsequent places, an individual wants to visit helps in identifying potential ride-sharing partners.

The current state-of-the-art to solve these prediction and labeling tasks is the usage of machine-learning methods (Toch et al. 2018). The usual approach is to extract meaningful features from the available movement and context data, and to use them for training a classifier for label-prediction tasks or a regressor for predicting future mobility demand. Here, the random-forest algorithm (Breiman 2001) is especially worth mentioning, as it is very robust with regard to the distribution of the input data, has generally a very good performance, and does not require extensive hyper-tuning of parameters.

An important research direction is to create spatially aware machine-learning methods (Gilardi and Bengio 2000; Hengl et al. 2018). One problem is that generalpurpose machine-learning algorithms do usually not consider spatial dependencies (e.g., spatial autocorrelation present in the input or output data; Cracknell and Reading 2014). Another recent research direction is to avoid the explicit feature extraction step altogether, because it usually implies the assumption of independent and identically distributed data. An alternative is the use of neural networks and learning feature maps directly from the data. However, here, it is often difficult to find a meaningful data representation that is suitable for neural networks. Possible representations are image representations (Chen et al. 2016a) or more recently graph representations (Martin et al. 2018).

\subsubsection{Studies}

In practice, studies based on tracking data are scarce and usually not publicly available. The most important reason for this is that personal tracking data are extremely privacy sensitive (Keßler and McKenzie 2018). This implies that on the one hand, it is difficult to find participants who are willing to share their geodata due to privacy concerns, and on the other hand, that datasets are unavailable for other research groups once they were collected. Resulting from this situation, there are two types of mobility studies: user studies based on participants that were recruited for the purpose of the study by a research group, and mobility studies based on data that were already collected for different purposes and contained the locations of users as a byproduct. The first type of study are also called active-tracking studies because users in these studies commonly provide feedback that can be used to label the data and to answer the underlying research questions. The second type of study is called passive-tracking studies because users are commonly unaware that they participate in a study and that their location is collected passively in the background without any possibility for the user to provide feedback. Some notable examples of mobility studies based on passive-tracking data sets include:

Brockmann et al. (2006) were among the first to use already-collected data (sightings of dollar bills from www.wheresgeorge.com) that contained information about human mobility as a byproduct. The analysis of this dataset with more than a million 
displacements uncovered fundamental statistical properties of human movement, such as a power-law distribution of traveling distances.

González et al. (2008) developed an early large mobility study based on CDR data collected for billing purposes by the mobile-phone provider, which also allowed for the reconstruction of human mobility patterns. These data allowed one to analyze the movement of individual persons over a time span of six months and revealed a high spatio-temporal regularity of human movement patterns.

Both studies are early representatives of large-scale empirical studies and are rather descriptive and general. Studies in later years became more specific:

Hasan et al. (2013) used data from smart cards utilized in public transportation systems to specifically analyze human mobility within a city. Among other results, this study reproduced the already known general mobility characteristics in an urban setting.

Yuan and Raubal (2016a) used CDR data that were enriched with demographic information to empirically analyze the spatial distribution of different demographic groups within a city.

Clemente et al. (2018) used credit card records in combination with CDR data from the same users to analyze urban mobility. This allowed them to cluster the users utilizing the semantically rich credit-card data and to interpret these clusters spatially using the CDR data.

The second type of study is significantly different as it involves only a small number of people but with very detailed data about these persons:

Eagle and Pentland (2006) conducted one of the first larger studies using mobile phones as wearable sensors. They collected information such as call logs, Bluetooth proximity data, and the current cell phone tower ID as a proxy for location. The goal of the study was to study not the mobility of the participants but rather their social interactions. This so-called reality-mining dataset is one of the first publicly available datasets that includes tracking data.

Zheng et al. (2008) introduced GeoLife, one of the first large GPS tracking studies, with 65 users being tracked for varying timespans within a ten-month period. These data were used to analyze individual mobility patterns. This dataset is publicly available and can be used for research purposes.

Alessandretti et al. (2018) used different publicly available datasets such as the reality-mining dataset and proprietary datasets such as the CNS dataset from Stopczynski et al. (2014) to show that persons only have a limited number of regularly visited locations and that, while the locations change slowly over time, the total number of locations stays constant. 


\subsubsection{SBB Green Class (Multi-modal and Energy-Efficient Mobility)}

This section presents one case study in greater detail, the SBB Green Class pilot studies. In 2016 and 2017, the Swiss federal railways (SBB) carried out two large, one-year pilot tests of a MaaS concept. In these studies, customers received access to comprehensive mobility options for a fixed yearly fee. The first pilot study had 150 participants from Switzerland, who received a Swiss-wide public transport pass, a battery electric vehicle, a parking space at their local train station, and credit for carsharing and bikesharing services. The second pilot study had 50 participants and included an e-bike instead of the e-car. As part of the pilot study, all participants installed a tracking app on their phone and agreed to label the recorded and segmented GPS tracks with the user mode of transport and a high-level description of the trip purpose. The most interesting characteristic of the SBB Green Class pilot studies is a flat rate for mobility, where almost all costs are covered by the subscription fee, making it the first study of this size that can be used to test the impact of MaaS offers.

To evaluate the mobility behavior of the participants the tracking data had to be prepared using different preprocessing steps, such as the fusion of different data sources, imputation of missing labels, map matching, grouping movement into trips and tours, and the detection of anomalies. Subsequently the participants' mobility behavior could be compared to a pseudo-control group generated from the Swiss mobility and transport microcensus (MTMC). The most important results were:

- Especially the Green Class e-car pilot study participants traveled more than the average Swiss person and were particularly frequently multimodal. These differences can be partially explained by the SBB Green Class offer: on the one hand, there are available parking facilities near the railway station, which clearly promote combined travel, and on the other hand the lower marginal costs for mobility invite passengers to longer and more frequent journeys.

- A comparison with the control group revealed that the electric car primarily replaced journeys with a conventional vehicle; the proportion of train journeys differed only slightly between Green Class customers and the control group.

- The analysis of the longitudinal tracking data showed that the $\mathrm{CO}_{2}$ emissions of most participants decreased significantly shortly after the start of the project. This can primarily be attributed to the electric vehicle, which has lower average $\mathrm{CO}_{2}$ emissions than a car with a combustion engine (especially when taking into account the Swiss electricity mix). The overall development of the Green Class e-car users' $\mathrm{CO}_{2}$ emissions and the possible impact of a MaaS offer can be seen in Fig. 6.2.

- A result that is particularly noteworthy is that the e-car established itself in the mobility mix of the participants in the long term while primarily replacing the conventional car. 


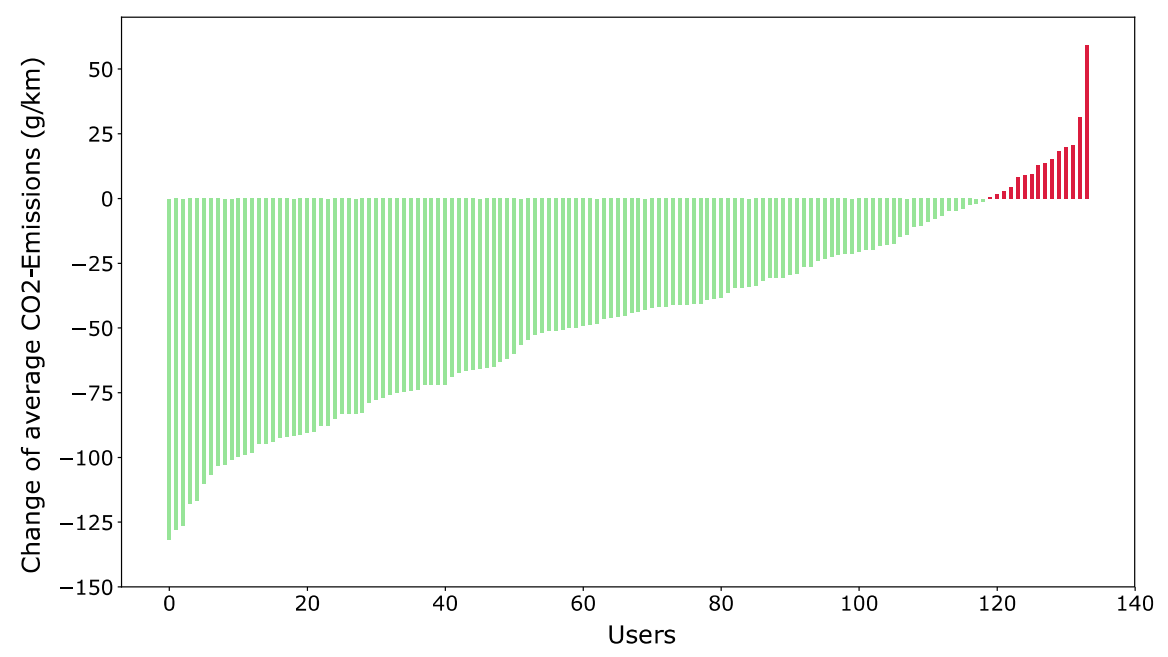

Fig. 6.2 Comparison of SBB Green Class 1 users' average $\mathrm{CO}_{2}$ emissions during a six-week preproject tracking phase and their emissions after they got access to the new mobility tools (public transport pass, e-car, etc.). Most participants (indicated in green) were able to reduce their $\mathrm{CO}_{2}$ emissions significantly and only few participants (indicated in red) increased their average $\mathrm{CO}_{2}$ emissions compared to before the project

\subsection{Behavioral Change and Sustainable Mobility}

It is often argued that making mobility ecologically sustainable requires a wide range of technical, institutional, and societal innovations, in particular in the short term (Banister 2008; Holden 2016; Kemp and Rotmans 2004). These innovations are related to the optimization and extension of public transport networks, to the electrification of car fleets alongside an increased renewable energy production, and also to various shifts in our use of mobility, for example from cars to alternative means of transport. The latter is commonly referred to as changing one's mobility behavior, and a substantial body of research concerns the effects of mobility behavior changes on a large scale (Bucher et al. 2019; Taniguchi and Fujii 2007), how ICT impacts people in their mobility planning and choices (Chen et al. 2016b; Cohen-Blankshtain and Rotem-Mindali 2016), how persuasive technologies can be used to nudge people toward certain desired behaviors (Gabrielli et al. 2014; Weiser et al. 2016), and how and where critical support infrastructure should be built to maximize its impact on mobility behavior (Buffat et al. 2018; Huétink et al. 2010). Here, we will focus on the potentials of novel geospatial and persuasive technologies alongside contextualized and personalized computational methods to help people travel sustainably. 


\subsubsection{Motivation}

Behavior is strongly driven by motivation, which in turn arises from two groups of base needs (Deci and Ryan 2004; Reeve 2014): Psychological needs form the most innate group and include the desire for autonomy, competence and relatedness. They describe the facts that humans like to be in control of their actions, that these actions must be challenging yet doable, and that people need to interact with others within meaningful relationships. Social needs are similarly about the cultivation of relationships but are learned over the course of our lives. They encompass achievement, affiliation, intimacy, and a desire for leader- and follower-ship.

Individual actions (such as choosing a particular mode of transport) are usually spurred by either external or internal motivational sources. External sources include monetary incentives, rewards, or simply promises by other people. In stark contrast, intrinsic motivation is generated by one's own goals, expectations, beliefs, and perceptions. At its core is the perception we have of ourselves, subconsciously built by inspecting the effects of our behavior on other people. Based on this, we develop attitudes and beliefs, on which we rely when formulating certain goals or building expectations. Intrinsic motivation correlates with the satisfaction of the above-mentioned base needs (Van den Broeck et al. 2016). If a human does not manage to live up to his or her core beliefs, a state of cognitive dissonance is entered, which forms a strong internal motivational source that can be used to induce behavior change.

Such a change of behavior can be modeled using the trans-theoretical model (Prochaska and Velicer 1997). On a high level, we can classify behavior change into two phases: discovery and maintenance (Li et al. 2011). The trans-theoretical model splits discovery into a pre-contemplation, a contemplation, and a preparation phase, which are characterized by a transition from being unaware of a certain behavior to starting to form plans on how to change it. The transition into maintenance is performed once a person starts taking actions, which are prompted by triggers, for example, receiving a notification about an upcoming appointment (Fogg 2009). After reaching a certain level of competence, people have to be kept from relapsing until the behavior is truly internalized and a new habit is formed. Smart geographic ICT must thus be aware of the different motivational factors and phases that influence individuals in varying ways and provide adapted support for people in different circumstances and contexts.

\subsubsection{Detecting and Supporting Behavioral Change}

A substantial amount of research focuses on using ICT to detect and identify activities related to movement and mobility (Feng and Timmermans 2013; Gong et al. 2012; Montini et al. 2014), in particular the motives for traveling somewhere as they heavily influence transport-mode choices. This identification of activities and transport modes becomes increasingly accurate as researchers get easier access to 
large ground-truth datasets that can be effectively used for machine learning and thus automated inference at scale.

Once the activities are known, their change over time can be analyzed to detect sudden or gradual changes in behavior and support users adequately throughout different motivational stages. Jonietz and Bucher (2018) continuously mined trajectories with the aim of identifying behavioral patterns and anomalies. They summarized daily and weekly mobility usage by computing characteristic features; for example, the number of trips taken or the total distance traveled with a certain mode of transport. An anomalous deviation of these features from one week to another can indicate a transition from one phase of behavior change to another and should be reflected within the supporting ICT. Additionally, identifying people in similar behavioral transition phases can be used for analytical purposes or to target individual groups with specific incentives (Zhao et al. 2019).

Depending on the motivational phase, people have different needs for support: someone (pre-)contemplating change is well served by information about the existence of alternative transport options; someone taking action requires external motivators and well-timed and appropriate triggers (Weiser et al. 2015). If a trigger manages to increase our motivation (e.g., by giving additional external rewards) or to decrease the difficulty of the action (e.g., by providing a meaningful sustainable mobility alternative), a user is much more likely to exhibit the desired behavior (Fogg 2009). To provide alternative mobility plans, ICT has to generate and evaluate them, taking into account sustainability as well as the user's context (e.g., the planned activity at the destination, or past and future trips). Based on a wealth of (multi-modal) transport planning systems (Bast et al. 2016), heuristic methods (Bucher et al. 2017), and approaches based on previously recorded movement (Arentze 2013; Campigotto et al. 2016) were developed to generate meaningful routes. The resulting alternatives are scored using the primary feature of interest, e.g., the total $\mathrm{CO}_{2}$ emissions, the distance, or the duration.

An often employed persuasive method is gamification, i.e., using game design elements in non-game contexts (Deterding et al. 2011). Gamification can be used as an external source of motivation by employing mechanisms such as feedback, rewards, challenges, competition, or cooperation (Weiser et al. 2015). These should follow a set of general design principles, such as offering meaningful suggestions, providing guidance, supporting user choices, or personalizing experiences. It needs to be noted that the use of common gamification elements for feedback on mobility behavior is not as straightforward as in other domains. As mobility is highly individual, simply offering rewards for taking the bicycle to work might be completely unfeasible for some while extremely easy for others. Similarly, rewarding points for taking public transport may lead to people trying to travel more, while the most ecologically friendly choice would likely be not to travel at all (Froehlich et al. 2009). 


\subsubsection{Studies}

Among the well-known early studies on the effects of persuasive ICT on mobility, choices and behavior are applications that feature a combination of movement tracking and technology-assisted feedback, commonly by showing users the impact of the $\mathrm{CO}_{2}$ emissions caused by their trips (Anagnostopoulou et al. 2018; Gössling 2018). UbiGreen (Froehlich et al. 2009) uses a combination of a mobile sensing platform, GSM cell tower localization, and information entered by users to record mobility patterns. It features a visual representation involving either a tree or an iceberg that indicates the effect of trips taken during a week. While there was no quantitative analysis of behavior change performed (due to the small sample size of 14 people and the short tracking duration of three weeks), interview responses demonstrated the viability of such eco-feedback applications. Similarly, MatkaHupi (Jylhä et al. 2013), tripzoom (Bie et al. 2012), the THELMA project (Bauer et al. 2016), or the Streetlife EU project (Kazhamiakin et al. 2015) featured smartphone applications that were used both as a tracker as well as for providing feedback to the mobility consumer.

Typically, these studies were performed with a smaller sample of participants (approximately 10-50) over the course of up to two months (Anagnostopoulou et al. 2018). Recently, several studies have tried to replicate their results with larger samples over longer periods of time. Research by Semanjski et al. (2016) involved a sixmonth data collection and intervention period with 3400 participants. During this time, movement data were collected and feedback given via a Web platform. Their results showed that eco-feedback can be used to initiate behavioral changes but the outcomes vary depending on the attitudinal profiles. Ebermann and Brauer (2016) similarly enrolled 248 participants to use a Web site during a three-week period and explored the influence of different goals ("self-exploration," "competition," "climate protection," etc.) on the use of various gamification elements. An additional large body of work emphasizes the use of persuasive technologies to improve personal health - which often leads to more ecologically sustainable travel behavior as well. Consolvo et al. (2008) explored the potential of early smartphones in combination with mobile sensing platforms to promote healthy lifestyles. Similarly, Harries et al. (2013) enrolled 152 participants for their study that used an app to promote walking behavior. They found that the app manages to increase the step count by around 64\%, but that comparative social feedback did not improve this value.

The latter also indicates that not all persuasive strategies work well in a mobility context. Gabrielli et al. (2014) summarize these challenges associated with inducing a mobility behavior change for more sustainable future urban mobility. They found that changing mobility behavior is a lengthy process and that it is very difficult to find motivational features that engage a wide range of users. In contrast to the personal health domain, collective mechanisms (i.e., social influence) tend to have a stronger influence on behavior than individual ones. Their findings corresponded to research by Nicholson (2012) and Weiser et al. (2015), who stressed that eco-feedback must be timely and meaningful. 


\subsubsection{GoEco!}

For a more in-depth account of a study targeting the change of mobility behavior, the example of GoEco! is chosen (Cellina et al. 2019). In contrast to previous studies, GoEco! targeted around 200 people from two diverse geographic regions; they were asked to participate in the experiment over the duration of a year. Within this year, three periods were chosen during which participants had to install an application on their smartphone that would simply record their movement in the first phase, give them additional eco-feedback (using gamification elements) in the second phase, and resort back to simple movement tracking for the third one (to determine potential long-term effects of the intervention in the second phase; Cellina et al. 2019).

The application used a naïve Bayes classifier to identify transport modes from several features, such as travel speed, journey distance, or the distance to public transport stops in the vicinity (Bucher et al. 2019). This transport-mode identification was then given to users for verification, after which several potential (and feasible) alternatives were computed for each trip. These alternatives were presented as feedback to people, together with an assessment of potential $\mathrm{CO}_{2}$ emission reductions stemming from transitions to different transport modes. In addition, the gamified feedback included personal goals, weekly challenges, badges as rewards for desirable behavior (e.g., taking the bicycle to work, or completing a certain challenge), and a leaderboard that ranked people according to the number of badges they collected (Fig. 6.3; Cellina et al. 2019b).

Studying the long-term effects, it was found that people in rural areas changed their behavior on systematic routes. This was partially due to the selection of participants, who came from the city of Zürich (where people are often already eco-friendly travelers due to artificially created impediments for car drivers) and the canton of Ticino (where public transport is less developed, and the private car is the primary means of transport). The fact that people changed their behavior on systematic routes (e.g., from home to work and back) is likely due to having more options on those (as

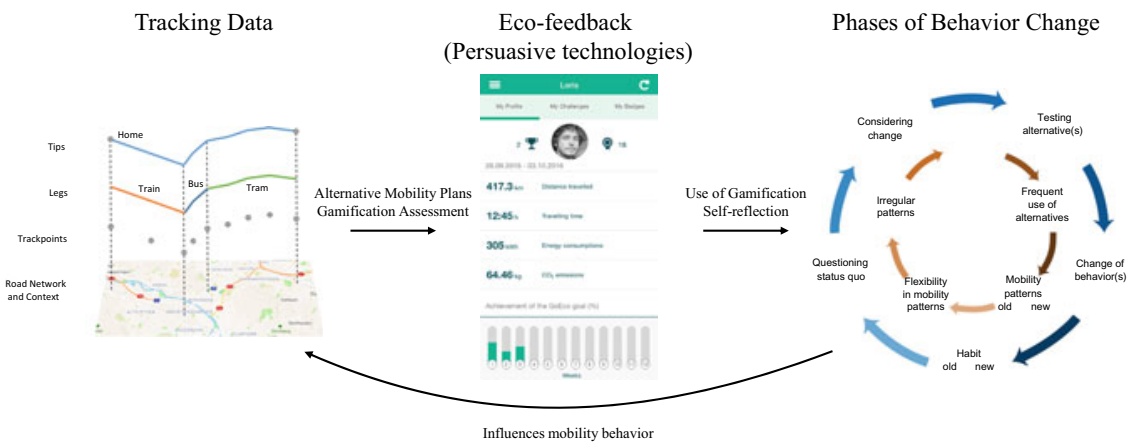

Fig. 6.3 Starting from movement and mobility tracking data, different mobility plans are evaluated, based on which gamified feedback is given. Users interpret and utilize the feedback differently depending on the phase of behavior change, which is reflected in the tracking data again 
one is potentially less restricted by context, such as the need to drive the whole family or carry shopping goods) and due to only having to find good alternatives a limited number of times (in contrast to non-systematic routes, where a suitable alternative has to be searched for every time).

\subsection{Mobile Decision Making}

Mobile geospatial technologies support people in their location-based decisionmaking, and at the same time acquire spatial big data, which can be utilized for urban planning and the enhancement of urban infrastructure resilience (Heinimann and Hatfield 2017). Mobile location-based decision-making encompasses a variety of spatio-temporal constraints, which relate not only to people's spatio-temporal behavior in large-scale space (Kuipers and Levitt 1988) but also to their interaction with mobile devices, and perceptual, cognitive, and social processes (Raubal 2015). People often need to make fast decisions on the spot, which requires both fast access to spatial memory and immediate system responsiveness. Furthermore, mobile devices such as mobile phones limit the communication process to their users, for example through small screen size, which makes it challenging to present information to someone on the move (Montello and Raubal 2012).

\subsubsection{Mobile Eye-Tracking and Gaze-Based Interaction}

As described earlier, geosmartness is also enabled by novel interaction modalities and paradigms, and one of these concerns gaze-based interaction. Gaze-based interaction is made possible by eye-tracking technology, and it is regarded as a particularly efficient and intuitive interaction modality (Majaranta and Bulling 2014), especially when interacting with space and visual-spatial representations (Kiefer et al. 2017). In explicit gaze-based interaction, the user deliberately triggers an interaction by looking at a certain position in the stimulus, whereas implicit gaze-based interaction refers to the automatic interpretation of eye movements for recognizing cognitive states, such as search activities on maps.

The ability to track gaze movements with eye-tracking technology allows measuring the current point of regard on a specific stimulus. There exist remote and mobile eye-tracking devices, and nowadays, most of them are video-based corneal reflection systems (Duchowski 2017). Mobile eye trackers measure a person's visual attention on a stimulus in the wild instead of the laboratory. The basic recordings are called gazes, and it is generally assumed that perception takes place only if gaze remains almost still for a minimum amount of time. Gazes are therefore often aggregated spatio-temporally to fixations. A transition between two fixations is called a saccade, which is caused by a rapid movement of the eye. Eye-tracking data can be used for investigating cognitive processes, such as self-localization during 
wayfinding (Kiefer et al. 2014), for activity recognition (Kiefer et al. 2013), and as input for gaze-based assistants. Many eye-tracking systems allow for real-time data access, which is the principle behind such gaze-assistive systems.

\subsubsection{Personalized Gaze-Based Decision Support}

Urban mobility and navigation of the future will become more complex for people due to the variety of combined transport modes offered by mobility-as-a-service options, increased environmental complexity (especially in megacities), and the multifaceted decision-making process of how to engage in sustainable mobility. Smart city environments, as described here, in combination with gaze-assistive systems, will allow personalized navigation support for their users.

Nowadays, navigation instructions are typically displayed as turn-by-turn instructions on a digital map presented on small mobile screens (Hirtle and Raubal 2013). Visual attention switches between display and environment can lead to high cognitive load (Bunch and Lloyd 2006) and distraction, such as in busy traffic situations. These problems can be avoided by utilizing gaze-based interaction concepts. An example is GazeNav (Fig. 6.4), which enables gaze-based interaction for pedestrian navigation (Giannopoulos et al. 2015). Gaze is utilized to inform the wayfinder whether the road that he or she is gazing at is the correct one to follow. To use this system, the user wears mobile eye-tracking glasses, which capture the current point of regard. When a decision point with different options is approached, the user starts to examine the possible ones to follow. At the moment when the user's gaze is aligned with the correct street, the system automatically provides feedback to convey this, for example through a vibrotactile belt or, more effectively, its combination with gaze information (Gkonos et al. 2017). Systems for real-time gaze tracking in outdoor environments, which map the gazes from a mobile eye tracker to a georeferenced view using computer vision methods, allow for such personalized gaze-based decision support (Anagnostopoulos et al. 2017).

The example of GazeNav illustrates how novel interaction modalities will impact our spatio-temporal decision-making in the future, leading to more personalized

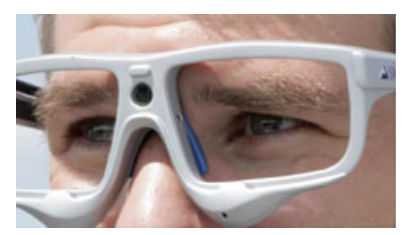

Gaze input

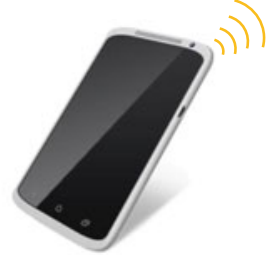

Navigation service

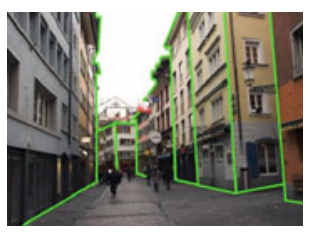

Model of surroundings

Fig. 6.4 Gaze-based pedestrian navigation 
information that can facilitate and improve people's decision processes. In addition, such technologies will also provide an enormous amount of spatial big data, in this case user-behavior data, which can be utilized by both the private and public sectors to improve old services and offer new ones. This implies that our locations will be shared with a multitude of different services, and therefore, the protection of geoprivacy in combination with other types of personal information will become an even more important issue in smart city environments (Keßler and McKenzie 2018; see Chap. 32).

\subsection{Conclusions and Future Work}

The ever-increasing urban mobility and transport of people has led to an increase of greenhouse-gas emissions and traffic jams. In this chapter, we demonstrated how geosmartness, a combination of novel spatial-data sources, computational methods, and geospatial technologies made possible through major advances in ICT helps to make urban mobility of the future more sustainable and personalized. On the one hand, novel movement-analytics methods including machine learning can be applied to massive volumes of tracking and context data, in order to make short- and longer-term predictions of transportation network states. This will help to optimize future states of the mobility system and to create flexible and personalized mobility offers. An overview of recent mobility studies and SBB Green Class, a detailed case study of multi-modal and energy-efficient mobility, served as examples. On the other hand, mobility-pattern analysis will help detect people's behavioral changes, and the impact of their travel habits and alternative travel modes, which in turn should pave the way toward more sustainable forms of transport. Sustainable urban mobility will be one contributor to the reduction of $\mathrm{CO}_{2}$ emissions in the future. We introduced methods for detecting and supporting behavioral change, related studies, and GoEco! as a concrete study targeting the change of mobility behavior through tracking data analysis and eco-feedback. Finally, from a user perspective people must also be directly supported in their complex mobile decision making. We proposed mobile eye tracking as a novel data source, which allows personalized gaze-based decision support in urban navigation. GazNav illustrated how gaze-based pedestrian navigation facilitates people's decision making based on the integration of gaze input, a navigation service, and a representative model of the environment.

Further research is necessary in all three of the discussed aspects of geosmartness, that is, spatial big data, spatio-temporal analysis methods, and geographic information technologies, in order to achieve a fully personalized and sustainable urban mobility of the future. For various states it will be important to have true real-time data from different sources-for example tracking, context, and socialmedia data-available, in order to evaluate a particular situation comprehensively and to detect the causes of a potential problem. The sheer data volume, and data integration and accuracy issues present obvious challenges. From a data analysis 
perspective, most machine-learning methods do not account for spatial autocorrelation; therefore, further research on how to make machine-learning methods spatially aware is required. In addition, most machine-learning models come as black boxes, which hinders interpretability and explanation of results. Machine-learning model interpretability is therefore a pressing issue (Hohman et al. 2019). Finally, future advancements in the area of urban informatics will continue to be technology driven. We expect novel geographic information technologies that will enhance both urban system evaluations and predictions, as well as mobile decision-making support for the individual user.

\section{References}

Alessandretti L, Sapiezynski P, Sekara V, Lehmann S, Baronchelli A (2018) Evidence for a conserved quantity in human mobility. Nat Hum Behav 2(7):485. https://doi.org/10.1038/s41 562-018-0364-x

Anagnostopoulos V, Havlena M, Kiefer P, Giannopoulos I, Schindler K, Raubal M (2017) Gazeinformed location-based services. Int J Geogr Inf Sci 31(9):1770-1797

Anagnostopoulou E, Bothos E, Magoutas B, Schrammel J, Mentzas G (2018) Persuasive technologies for sustainable mobility: state of the art and emerging trends. Sustainability 10(7):2128

Arentze TA (2013) Adaptive personalized travel information systems: a Bayesian method to learn users' personal preferences in multimodal transport networks. IEEE Trans Intell Transp Syst 14(4): 1957-1966

Banister D (2008) The sustainable mobility paradigm. Transp Policy 15(2):73-80

Banister D (2011) Cities, mobility and climate change. J Transp Geogr 19(6):1538-1546. https:// doi.org/10.1016/j.jtrangeo.2011.03.009

Bast H, Delling D, Goldberg A, Müller-Hannemann M, Pajor T, Sanders P, Wagner D, Wer-neck R (2016) Route planning in transportation networks. In: Algorithm engineering. Springer, pp 19-80

Batty M, Axhausen K, Giannotti F, Pozdnoukhov A, Bazzani A (2012) Smart cities of the future. Eur Phys J Spec Top 214(1):481-518. https://doi.org/10.1140/epjst/e2012-01703-3

Bauer C, Cox B, Heck T, Hirschberg S, Hofer J, Schenler W, Simons A, Del Duce A, Althaus H-J, Georges G (2016) Opportunities and challenges for electric mobility: an interdisciplinary assessment of passenger vehicles. Final report of the THELMA project in co-operation with the Swiss Competence Center for Energy Research "Efficient technologies and systems for mobility". Technical Report. ETH, Zürich

Bie J, Bijlsma M, Broll G, Cao H, Hjalmarsson A, Hodgson F, Holleis P, van Houten Y, Jacob K, Koolwaaij J (2012) Move better with tripzoom. Int J Adv Life Sci 4(3):125-135

Boulouchos K, Cellina F, Ciari F, Cox B, Georges G, Hirschberg S, Hoppe M, Jonietz D, Kannan R, Kovacs N, Küng L, Michl T, Raubal M, Rudel R, Schenler W (2017) Towards an energy efficient and climate compatible future Swiss transportation system. Working paper. SCCER Mobility

Breiman L (2001) Random forests. Mach Learn 45(1):5-32. https://doi.org/10.1023/A:101093340 4324

Brimicombe A, Li B (2009) Location-based services and geo-information engineering. WileyBlackwell, Chichester, UK

Brockmann D, Hufnagel L, Geisel T (2006) The scaling laws of human travel. Nature 439(7075):462-465. https://doi.org/10.1038/nature04292

Bucher D, Jonietz D, Raubal M (2017) A heuristic for multi-modal route planning. Progress in location-based services 2016. Springer, Berlin, pp 211-229 
Bucher D, Mangili F, Cellina F, Bonesana C, Jonietz D, Raubal M (2019) From location tracking to personalized eco-feedback: a framework for geographic information collection, processing and visualization to promote sustainable mobility behaviors. Travel Behav Soc 14:43-56

Buffat R, Bucher D, Raubal M (2018) Using locally produced photovoltaic energy to charge electric vehicles. Comput Sci Res Dev 33(1-2):37-47

Bunch R, Lloyd R (2006) The cognitive load of geographic information. Prof Geogr 58(2):209-220

Campigotto P, Rudloff C, Leodolter M, Baue D (2016) Personalized and situation-aware multimodal route recommendations: the FAVOUR algorithm. IEEE Trans Intell Transp Syst 18(1):92-102

Caragliu A, Del Bo C, Nijkamp P (2011) Smart cities in Europe. J Urban Technol 18(2):65-82. https://doi.org/10.1080/10630732.2011.601117

Cellina F, Bucher D, Mangili F, Simão J, Rudel R, Raubal M (2019) A large scale, app-based behaviour change experiment persuading sustainable mobility patterns: methods, results and lessons learnt. Sustainability 11(9):2674. https://doi.org/10.3390/su11092674

Cellina F, Bucher D, Simão J, Rudel R, Raubal M (2019) Beyond limitations of current behaviour change apps for sustainable mobility: insights from a user-centered design and evaluation process. Sustainability 11(8):2281. https://doi.org/10.3390/su11082281

Chen C, Ma J, Susilo Y, Liu Y, Wang M (2016) The promises of big data and small data for travel behavior aka human mobility analysis. Transp Res Part C: Emerg Technol 68:285-299

Chen Q, Song X, Yamada H, Shibasaki R (2016) Learning deep representation from big and heterogeneous data for traffic accident inference. Paper presented at the Thirtieth AAAI Conference on Artificial Intelligence

Clemente R, Luengo-Oroz M, Travizano M, Xu S, Vaitla B, González M (2018) Sequences of purchases in credit card data reveal lifestyles in urban populations. Nat Commun 9(1):3330. https://doi.org/10.1038/s41467-018-05690-8

Cohen-Blankshtain G, Rotem-Mindali O (2016) Key research themes on ICT and sustainable urban mobility. Int J Sustain Transp 10(1):9-17

Consolvo S, McDonald D, Toscos T, Chen M, Froehlich J, Harrison B, Klasnja P, LaMarca A, LeGrand L, Libby R (2008) Activity sensing in the wild: a field trial of ubifit garden. Paper presented at the SIGCHI Conference on Human Factors in Computing Systems

Cracknell MJ, Reading AM (2014) Geological mapping using remote sensing data: a comparison of five machine learning algorithms, their response to variations in the spatial distribution of training data and the use of explicit spatial information. Comput Geosci 63:22-33. https://doi. org/10.1016/j.cageo.2013.10.008

Deci EL, Ryan RM (2004) Handbook of self-determination research. University of Rochester Press, Rochester, NY

Deterding S, Dixon D, Khaled R, Nacke L (2011) From game design elements to gamefulness: defining "gamification". Paper presented at the 15th International Academic MindTrek Conference: Envisioning Future Media Environments

Duchowski A (2017) Eye tracking methodology: theory and practice, 3rd edn. Springer, London

Eagle N, Pentland A (2006) Reality mining: sensing complex social systems. Pers Ubiquit Comput 10(4):255-268. https://doi.org/10.1007/s00779-005-0046-3

Ebermann C, Brauer B (2016) The role of goal frames regarding the impact of gamified persuasive systems on sustainable mobility behavior. Paper presented at the 24th European Conference on Information Systems

Elliott A, Urry J (2010) Mobile lives London. Routledge, London

Feng T, Timmermans HJ (2013) Transportation mode recognition using GPS and accelerometer data. Transp Res Part C: Emerg Technol 37:118-130

Fogg B (2009) A behavior model for persuasive design. Paper presented at the 4th international conference on persuasive technology

Froehlich J, Dillahunt T, Klasnja P, Mankoff J, Consolvo S, Harrison B, Landay J (2009) UbiGreen: investigating a mobile tool for tracking and supporting green transportation habits. Paper presented at the SIGCHI conference on human factors in computing systems 
Gabrielli S, Forbes P, Jylhä A, Wells S, Sirén M, Hemminki S, Nurmi P, Maimone R, Masthoff J, Jacucci G (2014) Design challenges in motivating change for sustainable urban mobility. Comput Hum Behav 41:416-423

Gasco-Hernandez M (2018) Building a smart city: lessons from Barcelona. Commun ACM 61(4):50-57

Giannopoulos I, Kiefer P, Raubal M (2015) GazeNav: Gaze based pedestrian navigation. Paper presented at MobileHCI, 17th International conference on human-computer interaction with mobile devices and services, Copenhagen, Denmark

Gilardi N, Bengio S (2000) Local machine learning models for spatial data analysis. J Geogr Inf Decis Anal 4(1):11-28

Gkonos C, Giannopoulos I, Raubal M (2017) Maps, vibration or gaze? Comparison of novel navigation assistance in indoor and outdoor environments. J Location Based Serv 11(1):29-49. https:// doi.org/10.1080/17489725.2017.1323125

Gong H, Chen C, Bialostozky E, Lawson CT (2012) A GPS/GIS method for travel mode detection in New York City. Comput Environ Urban Syst 36(2):131-139

González MC, Hidalgo CA, Barabási AL (2008) Understanding individual human mobility patterns. Nature 453(7196):779-782. https://doi.org/10.1038/nature06958

Goodchild M (1992) Geographical information science. Int J Geogr Inf Sci 6(1):31-45

Gössling S (2018) ICT and transport behavior: a conceptual review. Int J Sustain Transp 12(3):153164

Harries T, Eslambolchilar P, Stride C, Rettie R, Walton S (2013) Walking in the wild: using an always-on smartphone application to increase physical activity. Paper presented at the IFIP conference on human-computer interaction

Hasan S, Zhan X, Ukkusuri S (2013) Understanding urban human activity and mobility patterns using large-scale location-based data from online social media. In: Proceedings of the 2 nd ACM SIGKDD international workshop on urban computing

Hecht B, Schöning J, Erickson T, Priedhorsky R (2011) Geographic human-computer interaction. Paper presented at the CHI 11 Extended Abstracts on human factors in computing systems, Vancouver, BC, Canada

Heinimann H, Hatfield K (2017) Infrastructure resilience assessment, management and governance — state and perspectives. In: Linkov I, Palma-Oliveira J (eds) Resilience and risk—methods and application in environment, cyber and social domains. Springer, Berlin, pp 147-187

Hengl T, Nussbaum M, Wright MN, Heuvelink GB, Gräler B (2018) Random forest as a generic framework for predictive modeling of spatial and spatio-temporal variables. PeerJ 6:e5518

Hirtle S, Raubal M (2013) Many to many mobile maps. In: Raubal M, Mark D, Frank A (eds) Cognitive and linguistic aspects of geographic space: new perspectives on geographic information research. Springer, Berlin, Heidelberg, pp 141-157

Hohman F, Head A, Caruana R, DeLine R, Drucker S (2019) Gamut: a design probe to under-stand how data scientists understand machine learning models. Paper presented at the $\mathrm{CHI}$ conference on human factors in computing systems CHI 2019, 4-9 May 2019, Glasgow, UK

Holden E (2016) Achieving sustainable mobility: everyday and leisure-time travel in the EU. Routledge

Huétink FJ, van der Vooren A, Alkemade F (2010) Initial infrastructure development strategies for the transition to sustainable mobility. Technol Forecast Soc Chang 77(8):1270-1281

Jonietz D, Bucher D (2017) Towards an analytical framework for enriching movement trajectories with spatio-temporal context data. Paper presented at the 20th AGILE conference on geographic information science, 9-12 May 2017, Wageningen, The Netherlands

Jonietz D, Bucher D (2018) Continuous trajectory pattern mining for mobility behaviour change detection. In: Proceedings of the 14th international conference on location based services. Springer, Cham, Switzerland, pp 211-230

Jylhä A, Nurmi P, Sirén M, Hemminki S, Jacucci G (2013) Matkahupi: a persuasive mobile application for sustainable mobility. In: Proceedings of the 2013 ACM conference on pervasive and ubiquitous computing, pp 227-230 
Kazhamiakin R, Marconi A, Perillo M, Pistore M, Valetto G, Piras L, Avesani F, Perri N (2015) Using gamification to incentivize sustainable urban mobility. Paper presented at the 2015 IEEE first international smart cities conference ISC2.

Kemp R, Rotmans J (2004) Managing the transition to sustainable mobility. In: System innovation and the transition to sustainability: theory, evidence and policy, pp 137-167

Keßler C, McKenzie G (2018) A geoprivacy manifesto. Transactions in GIS 22:3-19. https://doi. org/10.1111/tgis. 12305

Kiefer P, Giannopoulos I, Raubal M (2013) Using eye movements to recognize activities on cartographic maps. Paper presented at the 21th SIGSPATIAL international conference on advances in geographic information systems, 9-12 Nov 2013, Orlando, FL, USA

Kiefer P, Giannopoulos I, Raubal M (2014) Where am I? Investigating map matching during selflocalization with mobile eye tracking in an urban environment. Trans GIS 18(5):660-686. https:// doi.org/10.1111/tgis. 12067

Kiefer P, Giannopoulos I, Raubal M, Duchowski A (2017) Eye tracking for spatial research: cognition, computation, challenges. Spatial Cogn Comput 17(1-2):1-19. https://doi.org/10.1080/138 75868.2016.1254634

Knoblauch RL, Pietrucha MT, Nitzburg M (1996) Field studies of pedestrian walking speed and start-up time. Transp Res Rec 1538(1):27-38. https://doi.org/10.1177/0361198196153800104

Krzyżanowski M, Kuna-Dibbert B, Schneider J (2005) Health effects of transport-related air pollution. WHO Regional Office Europe

Kuipers B, Levitt T (1988) Navigation and mapping in large-scale space. AI Mag 9(2):25-43

Kwok T, Kiefer P, Schinazi V, Adams B, Raubal M (2019) Gaze-guided narratives: adapting audio guide content to gaze in virtual and real environments. Paper presented at the CHI Conference on Human Factors in Computing systems CHI 2019, 4-9 May 2019, Glasgow, Scotland

Laube P (2014) Computational movement analysis. Springer International Publishing, New York, NY

Li I, Dey AK, Forlizzi J (2011) Understanding my data, myself: supporting self-reflection with ubicomp technologies. In: Proceedings of the 13th international conference on ubiquitous computing, pp 405-414

Li S, Dragicevic S, Castro F, Sester M, Winter S, Coltekin A, Pettit C, Jiang B, Haworth J, Stein A, Cheng T (2016) Geospatial big data handling theory and methods: a review and research challenges. ISPRS J Photogr Remote Sens 115:119-133

Long JA, Nelson TA (2013) A review of quantitative methods for movement data. Int J Geogr Inf Sci 27(2):292-318. https://doi.org/10.1080/13658816.2012.682578

Longley P, Goodchild M, Maguire D, Rhind D (2011) Geographic information systems and science, 3rd edn. Wiley, Chichester, UK

Majaranta P, Bulling A (2014) Eye tracking and eye-based human-computer interaction. In: Fairclough S, Gilleade K (eds) Advances in physiological computing. Springer, pp 39-65

Martin H, Bucher D, Suel E, Zhao P, Perez-Cruz F, Raubal M (2018) Graph convolutional neural networks for human activity purpose imputation. Paper presented at the modeling and decisionmaking in the spatiotemporal domain workshop at the 32nd conference on neural information processing systems NIPS, Montreal, Canada

Mirowski P, Grimes MK, Malinowski M, Hermann KM, Anderson K, Teplyashin D, Simonyan K, Kavukcuoglu K, Zisserman A, Hadsell R (2019) Learning to navigate in cities without a map. arXiv: 180400168 [cs]

Montello D (1993) Scale and multiple psychologies of space. In: Frank AU, Campari I (eds) Spatial information theory: theoretical basis for GIS. Springer, Heidelberg-Berlin, pp 312-321

Montello D, Raubal M (2012) Functions and applications of spatial cognition. In: Waller D, Nadel L (eds) Handbook of spatial cognition. American Psychological Association, Washington, DC, pp 249-264

Montini L, Rieser-Schüssler N, Horni A, Axhausen KW (2014) Trip purpose identification from GPS tracks. Transp Res Rec 2405(1):16-23 
Nam T, Pardo T (2011) Smart city as urban innovation: Focusing on management, policy, and context. Paper presented at the fifth international conference on theory and practice of electronic governance, Tallinn, Estonia

Nicholson S (2012) A user-centered theoretical framework for meaningful gamification. Paper presented at Games + Learning + Society

Prochaska JO, Velicer WF (1997) The transtheoretical model of health behavior change. Am J Health Promot 12(1):38-48

Ratti C, Claudel M (2016) The city of tomorrow: sensors, networks, hackers, and the future of urban life. Yale University Press, New Haven, CN

Raubal M (2015) Mobile cognition: balancing user support and learning. Paper presented at WMC 2015-workshop on mobile cognition: using mobile devices to enhance human cognition, MobileHCI '15: 17th international conference on human-computer interaction with mobile devices and services adjunct proceedings, Copenhagen, Denmark

Raubal M (2018) Cognition and geographic information technologies. In: Montello D (ed) Handbook of behavioral and cognitive geography. Edward Elgar, Cheltenham, UK, pp 197-210

Raubal M (2019) It's the spatial data science, stupid! Paper presented at the spatial data science symposium - "Setting the spatial data science agenda", Santa Barbara, California. Retrieved from https://spatial.ucsb.edu/eventfiles/SDSS2019/Bio-Position-Raubal.pdf

Raubal M, Wang S, Guo M, Jonietz D, Kiefer P (eds) (2018) Spatial big data and machine learning in GIScience. Workshop at GIScience 2018, Melbourne, Australia

Reeve J (2014) Understanding motivation and emotion. Wiley, New York, NY

Reitsma F (2012) Revisiting the 'Is GIScience a science?' debate or quite possibly scientific gerrymandering. Int J Geogr Inf Sci 27(2):211-221

Rudi D, Giannopoulos I, Kiefer P, Peier C, Raubal M (2016) Interacting with maps on optical head-mounted displays. Paper presented at Spatial User Interaction SUI, 15-16 Oct 2016, Tokyo, Japan

Sapiezynski P, Stopczynski A, Gatej R, Lehmann S (2015) Tracking human mobility using wifi signals. PLoS ONE 10(7):e0130824. https://doi.org/10.1371/journal.pone.0130824

Semanjski I, Lopez Aguirre A, De Mol J, Gautama S (2016) Policy 2.0 platform for mobile sensing and incentivized targeted shifts in mobility behavior. Sensors 16(7):1035

Sharif M, Alesheikh AA (2018) Context-aware movement analytics: implications, taxonomy, and design framework. Wiley Interdisc Rev Data Mining Knowl Discov 8(1):e1233. https://doi.org/ 10.1002/widm 1233

Stopczynski A, Sekara V, Sapiezynski P, Cuttone A, Madsen MM, Larsen JE, Lehmann S (2014) Measuring large-scale social networks with high resolution. PLoS ONE 9(4):e95978. https://doi. org/10.1371/journal.pone.0095978

Suel E, Polak JW, Bennett JE, Ezzati M (2019) Measuring social, environmental and health inequalities using deep learning and street imagery. Sci Rep 9(1):1-10. https://doi.org/10.1038/s41598019-42036-w

Taaffe E, Gauthier H, O’Kelly M (1996) Geography of transportation. Prentice Hall, Englewood Cliffs, NJ

Taniguchi A, Fujii S (2007) Promoting public transport using marketing techniques in mobility management and verifying their quantitative effects. Transportation 34(1):37

Toch E, Lerner B, Ben-Zion E, Ben-Gal I (2018) Analyzing large-scale human mobility data: a survey of machine learning methods and applications. Knowl Inf Syst. https://doi.org/10.1007/ s10115-018-1186-x

UN (2014) World urbanization prospects: 2014 revision, highlights. Retrieved from https://esa.un. org/unpd/wup/publications/files/wup2014-highlights.Pdf

Van den Broeck A, Ferris D, Chang C-H, Rosen C (2016) A review of self-determination theory's basic psychological needs at work. J Manag 42(5):1195-1229

Weiser P, Bucher D, Cellina F, Luca VD (2015) A taxonomy of motivational affordances for meaningful gamified and persuasive technologies. Paper presented at ICT4S 2015 
Weiser P, Scheider S, Bucher D, Kiefer P, Raubal M (2016) Towards sustainable mobility behavior: research challenges for location-aware information and communication technology. GeoInformatica 20:213-239. https://doi.org/10.1007/s10707-015-0242-x

Yuan Y, Raubal M (2016a) Analyzing the distribution of human activity space from mobile phone usage — an individual and urban-oriented study. Int J Geogr Inf Sci 30(8):1594-1621. https://doi. org/10.1080/13658816.2016.1143555

Yuan Y, Raubal M (2016b) Exploring georeferenced mobile phone datasets-a survey and reference framework. Geogr Compass 10(6):239-252. https://doi.org/10.1111/gec3.12269

Yuan Y, Raubal M, Liu Y (2012) Correlating mobile phone usage and travel behaviour-a case study of Harbin, China. Comput Environ Urban Syst 36(2):118-130

Zhao P, Bucher D, Martin H, Raubal M (2019) A clustering-based framework for understanding individuals' travel mode choice behavior. In: Kyriakidis P, Hadjimitsis D, Skarlatos D, Mansourian A (eds) Geospatial technologies for local and regional development. In: Pro-ceedings of the 22nd AGILE conference on geographic information science. Springer, Cham, Switzerland, pp 77-94

Zheng Y (2015) Trajectory data mining: an overview. ACM Trans Intel Syst Technol 6:1-41. https:// doi.org/10.1145/2743025

Zheng Y, Wang L, Zhang R, Xie X, Ma W (2008) GeoLife: managing and understanding your past life over maps. Paper presented at the ninth international conference on mobile data management

Zhong C, Batty M, Manley E, Wang J, Wang Z, Chen F, Schmitt G (2016) Variability in regularity: mining temporal mobility patterns in London, Singapore and Beijing using smart-card data. PLoS ONE 11(2):e0149222. https://doi.org/10.1371/journal.pone.0149222
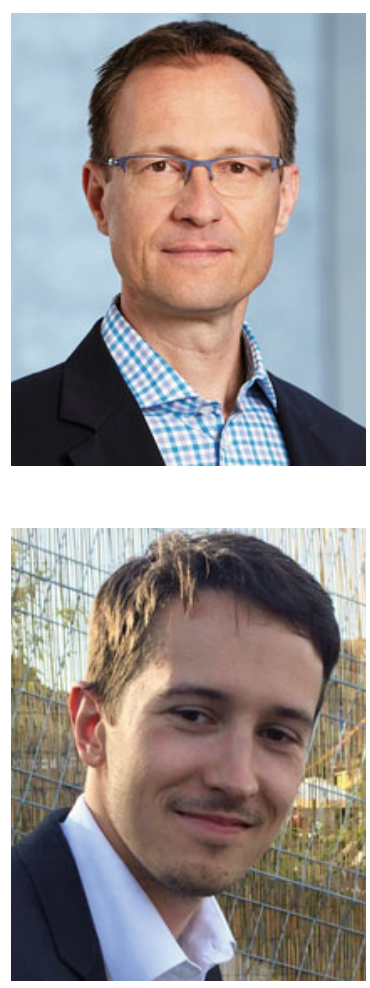

Martin Raubal is Professor of Geoinformation Engineering at ETH Zürich, Switzerland. He was a Co-chair of AGILE (Association of Geographic Information Laboratories in Europe) from 2014 to 2019. His research interests include Spatial Data Science, Location Based Services, spatial cognitive engineering, and mobile eye-tracking.

Dominik Bucher is a doctoral student in the group of Geoinformation Engineering at ETH Zürich, Switzerland. Within the Mobility Information Engineering Lab, he is working on methods to process and analyze human movement and mobility data with the aim of supporting people in reaching more sustainable behavior. 


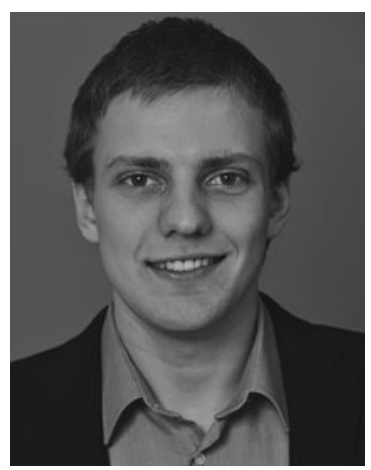

Henry Martin is working as a doctoral student in the Mobility Information Engineering Lab (MIE) at the Chair of Geoinformation Engineering at ETH Zürich. He is interested in applying modern data analysis methods to spatio-temporal problems and enable mobility as a driver towards a more sustainable energy system.

Open Access This chapter is licensed under the terms of the Creative Commons Attribution 4.0 International License (http://creativecommons.org/licenses/by/4.0/), which permits use, sharing, adaptation, distribution and reproduction in any medium or format, as long as you give appropriate credit to the original author(s) and the source, provide a link to the Creative Commons license and indicate if changes were made.

The images or other third party material in this chapter are included in the chapter's Creative Commons license, unless indicated otherwise in a credit line to the material. If material is not included in the chapter's Creative Commons license and your intended use is not permitted by statutory regulation or exceeds the permitted use, you will need to obtain permission directly from the copyright holder.

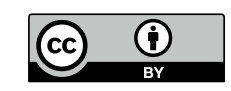

